\title{
Contribution of nonhydrodynamic interactions to the concentration dependence of the friction factor of the mutual diffusion coefficient ${ }^{\text {a) }}$
}

\author{
George D. J. Phillies \\ Department of Chemistry, The University of Michigan, Ann Arbor, Michigan 48109 \\ (Received 19 September 1980; accepted 31 October 1980)

\begin{abstract}
The mutual diffusion coefficient $D_{m}$ of a Brownian particle may be expressed as the ratio of a thermodynamic factor $K$ and a friction factor $f_{m}, K$ and $f_{m}$ both being dependent on the macroparticle concentration. The contribution to $f_{m}$ of direct (e.g., hard sphere, electrostatic) interactions is here estimated to first order in the macroparticle concentration. In contrast to our earlier calculation [J. Chem. Phys. 67, 4690 (1977)] in which the direct interaction contribution $\Delta f_{m}$ to the Stokes' Law drag coefficient was obtained from a fluctuation-dissipation equation. $\Delta f_{m}$ is here obtained directly from a mechanical argument based on the equations of motion of the macroparticles in solution. $\Delta f_{m}$ is explicitly evaluated for the case of particles
\end{abstract} \\ which interact through a weak Gaussian potential.
}

\section{INTRODUCTION}

The frictional force $-f v$ on a moving macroparticle has usually been interpreted in terms of hydrodynamic effects, the concentration dependence of the drag coefficient $f$ being taken to arise entirely from perturbations of the solvent flow around a probe macroparticle due to the other macroparticles in the solution. Direct interparticle interactions then affect $f$ in a secondary way; by altering the relative likelihood of different configurations of neighboring macroparticles, the direct interactions influence the probability of perturbations of the solvent flow around a probe macroparticle. Some years ago, $\mathrm{Mazo}^{1}$ showed that direct interactions between diffusing particles also can make an intrinsic contribution to $f$, as reflected by measured values ${ }^{2}$ for the tracer diffusion coefficient of sodiumlauryl sulphate micelles. More recently, this author argued $^{3}$ that, in the absence of hydrodynamic interactions, direct interactions should increase the Stokes' law drag coefficient $f_{S}$ of a macromolecule in concentrated solution.

The calculations of Refs. 1 and 3 are both based on fluctuation-dissipation type relations which equate a drag coefficient to a time integral over a force-force correlation function. While a relation such as

$$
f=\frac{1}{3 K_{B} T} \int_{0}^{t}\langle\mathbf{F}(0) \cdot \mathbf{F}(t)\rangle d t
$$

is a convenient starting point for a calculation, Eq. (1) and its usual derivations mask the casual chain leading from the movements of a probe macromolecule through the series of events which reposition the other macromolecules in the system into configurations in which $-f v$ is enhanced. Furthermore, Eq. (1) and the arguments based on it apply directly to the Stokes' law drag coefficient $f_{T}$ which is relevant to the tracer diffusion coefficient $D_{T}=K_{B} T / f_{T}$. For the mutual diffusion coefficient $D_{m}$ and its associated drag coefficient $f_{m}$, matters are more complex. Indeed, Weiss man ${ }^{4}$ argues that while the fluctuation-dissipation argument does apply to $f_{T}$, a more comprehensive analysis shows that there is not a direct interaction contribution to $f_{m}$.

\footnotetext{
a) This work supported in part by the National Science Foundation under Grant Che-7920399.
}

In this paper a novel direct mechanical procedure is used to estimate the effect of direct intermacromolecular interactions on $f_{m}$. In Sec. II, we re-examine the case of a macromolecule moving with constant velocity $v_{0}$, using the new procedure to rederive the central result of Ref. 3. Having demonstrated the procedure in a simple case, we proceed in Sec. III to estimate the (frequency dependent) contribution of direct interactions to $f_{m}$. Section IV discusses our results.

\section{DIRECT INTERACTIONS AND STOKES' LAW DRAG}

Reference 3 obtains a fluctuation-dissipation form for the direct contribution to $f_{s^{\circ}}$ Using the prescription (see Appendix)

$$
\begin{aligned}
& \int_{a_{k 0}} d\{N\} \exp [-\beta(W-A)]-\int d\{N\} \exp [-\beta(W-A)] \\
& \quad \times \exp \left(\sum_{i=1}^{N} \frac{a_{k 0} \exp \left(-i \mathbf{k} \cdot \mathbf{r}_{i}\right)}{\left\langle\left|a_{k}\right|^{2}\right\rangle}\right),
\end{aligned}
$$

(where $\mathbf{r}_{i}$ is the location of one of the $N$ macroparticles in the system, $W$ is their total potential energy, $A$ is the Helmholz free energy, $\int d\{N\}$ denotes an integral over all particle configurations, $\left\langle\left|a_{k}\right|^{2}\right\rangle$ is the ensemble average of the squared magnitude of the $k$ th spatial Fourier component of the macroparticle density, and $\int_{a_{k 0}} d\{N\}$ denotes a constrained integral limited to configurations for which

$$
\sum_{i=1}^{N} \exp \left(i \mathrm{k} \cdot \mathrm{r}_{1}\right)=a_{k 0},
$$

to replace the constrained integral with an unconstrained integral [Eq. (2.9)] of Ref. 3 becomes

$$
\begin{aligned}
\mathfrak{f}(\tau)= & \frac{-K_{B} T c_{0} \mathrm{v}_{0}}{(2 \pi)^{3}} \int d \mathrm{q} \frac{[h(q)]^{2}}{\left\langle\left|a_{\mathrm{q}}(0)\right|^{2}\right\rangle}\left(\mathrm{q} \cdot \hat{\mathrm{v}}_{0}\right)^{2} \\
& \times\left\{\left(\exp \left[\left(-i \mathrm{q} \cdot \mathrm{v}_{0}-\Gamma_{q}\right) \tau\right]-1\right) /\left(-i q \cdot \mathbf{v}_{0}-\Gamma_{\mathrm{q}}\right)\right\},
\end{aligned}
$$

where $g(r)$ is the macroparticle radial distribution function, $\Gamma_{q}$ is the relaxation time for macroparticle density fluctuations of wave vector $q$, and

$$
h^{(2)}(q)=\int d \mathrm{r} \exp (i \mathrm{q} \cdot \mathrm{r})[g(r)-1] .
$$


Using the numerical approximations of Ref. 3, except assuming $\Gamma_{q}=K_{B} T q^{2} / f\left\langle\left|a_{q}\right|^{2}\right\rangle$, one finds

$$
\mathbf{f}=-\mathbf{v}_{0} f c_{0} \int \frac{d \mathbf{k}}{(2 \pi)^{3}}\left(\hat{\mathbf{v}}_{0} \cdot \mathbf{k}\right)^{2}[h(k)]^{2},
$$

which result was previously obtained in Ref. 3 .

Our new argument considers a probe molecule in a suspension of similar particles. Initially $(t=0)$ the radial distribution of particles around the probe is spherically symmetric. The probe is then brought instantaneously to some (small) velocity $\mathrm{v}_{0}$. The distribution of particles around the probe attempts to track the motion of the probe. However, because the relaxation time of the suspension is not zero, the particles in the radial distribution function tend to lag be hind the probe, their positions being characterized by a distribution function $g(\mathbf{r} ; \Delta \mathrm{x}, t)$ which expresses the probability of funding a particle at $r$ relative to the probe given that the probe moved through $\Delta \mathbf{x}$ during the interval $(0, t) . g(\mathbf{r} ; \Delta \mathbf{x}, t)$ is not spherically symmetric; the asymmetry in $g$ produces a net retarding force on the probe. An argument related to this appears in the classic paper of Onsager and Fuoss ${ }^{5}$; these authors, however, explicitly state that the effect which they treat does not modify the mutual diffusion coefficient. We now estimate $g$ and compute the drag force which it produces.

Because of its symmetry, the initial $g_{\circ q}\left(r_{0}\right)$ for a particle at the origin can be expanded entirely in terms of cosine waves, namely,

$$
g_{\text {oq }}\left(\mathbf{r}_{0}\right)=1+\frac{1}{(2 \pi)^{3}} \int d q h(q) \cos \left(q \cdot r_{0}\right)
$$

In describing the distribution of particles around a probe located at $\Delta x$, one commonly discusses the symmetric $g_{\text {eq }}(\mathbf{r}), \mathbf{r}$ being measured from the probe. For the problem considered here, it is more convenient to expand $g_{0 q}$ in a coordinate system fixed in space. Defining $\mathbf{R}$ as the space-fixed coordinate and $\mathbf{r}$ as the particlefixed coordinate, $R=r+\Delta x$

$g_{\text {eq }}(\mathbf{R})=1+\frac{1}{(2 \pi)^{3}} \int d \mathrm{q} h(\mathrm{q})$

$x[\cos (\mathbf{q} \cdot \mathbf{R}) \cos (\mathbf{q} \cdot \Delta x)+\sin (\mathbf{q} \cdot \mathbf{R}) \sin (\mathbf{q} \cdot \Delta x)]$.

Equation (8) would always be exact if the solution adjusted instantaneously to the changing position of the probe. The behavior of a real system may be described by the expansion

$g(r ; \Delta \mathbf{x}, t)=1+\int \frac{d \mathrm{q}}{(2 \pi)^{3}}\left[a_{q}(t) \cos (\mathbf{q} \cdot \mathbf{R})+b_{q}(t) \sin (\mathbf{q} \cdot \mathbf{R})\right]$,

$a_{q}(t)$ and $b_{q}(t)$ being implicit functions of $\Delta x$. For $g(\mathbf{R})$ around a moving probe, the $a_{q}(t)$ and $b_{q}(t)$ do not achieve their limiting values $h(q) \cos (\mathbf{q} \cdot \Delta \mathbf{x})$ and $h(q) \sin (\mathrm{q} \cdot \Delta \mathbf{x})$.
Their relaxation rates are determined by diffusion; as suming that their regression is adequately described by a linear process, one finds

$$
\begin{aligned}
& \frac{d a_{q}(t)}{d t}=-\Gamma_{q}\left(a_{q}(t)-h(q) \cos [\mathbf{q} \cdot \Delta \mathbf{x}(t)]\right), \\
& \frac{d b_{q}(t)}{d t}=-\Gamma_{q}\left(b_{q}(t)-h(q) \sin [\mathbf{q} \cdot \Delta \mathbf{x}(t)]\right) .
\end{aligned}
$$

If we initially have an equilibrium ensemble of probes, at $t=0 a_{q}(0)=h(q)$ and $b_{q}(0)=0$;

$a_{q}(t)=h(q) e^{-\Gamma_{q} t}+\Gamma_{a} \int_{0}^{t} d s e^{-\Gamma_{q}(t-s)} h(q) \cos [q \cdot \Delta \mathbf{x}(s)]$

$b_{q}(t)=\Gamma_{q} \int_{0}^{t} d s e^{-\Gamma_{q}(t-s)} h(q) \sin [q \cdot \Delta \mathbf{x}(s)]$,

where, when $\Delta \mathbf{x}=0$, direct probe-particle interactions do not affect the temporal behavior of the $b_{q}(t)$. In this case, the probe-solution forces are spherically symmetric, tending equally to increase (or decrease) the concentration at points $+\mathbf{R}$ and $-\mathbf{R}$. However, the $b_{q}(t)$ are antisymmetric around the probe; forces having equal effects at $\pm \mathbf{R}$ change the $a_{a}(t)$ but have the wrong symmetry to couple to the $b_{Q}(t)$. Hydrodynamic interactions between the probe and its neighbors will affect $\Gamma_{q}$, in that an Oseen-type interaction will uniformly slow the motion of near neighbors of the probe, regardless of whether the motion is out of a region where $b_{q} \sin (q \cdot \mathbf{R})$ is positive or into a region where $b_{q} \sin (q \cdot \mathbf{R})$ is negative. If hydrodynamic interactions are ignored and clusters containing more than two particles are unimportant, the relaxation rates of the $a_{q}$ and the $b_{q}$ are the same. If these conditions are not satisfied, $\Gamma_{q}$ describes an aspect of the decay of the three-point density correlation function.

Given that the density of particles around the probe is not spherically symmetric, the nonvanishing force on the probe is

$$
\langle\mathbf{F}\rangle=c_{0} \int d \mathbf{r} g(\mathbf{r} ; \Delta \mathbf{x}, t) \frac{\partial W}{\partial r} \hat{\mathbf{r}} .
$$

Using Eqs. (9) and (11) for $g$, one has

$$
\begin{aligned}
\langle\mathbf{F}\rangle= & c_{0} \int d \mathbf{r}\left[1+\frac{1}{(2 \pi)^{3}} \int d \mathrm{q}\left\{\operatorname { c o s } ( \mathbf { q } \cdot \mathbf { R } ) \left[h(q) e^{-\Gamma_{q} t}\right.\right.\right. \\
& \left.+\Gamma_{q} \int_{0}^{t} d s \exp \left[-\Gamma_{q}(t-s)\right] h(q) \cos (\mathrm{q} \cdot \Delta \mathbf{x}(s))\right] \\
& \left.+\sin (\mathrm{q} \cdot \mathbf{R}) \Gamma_{q} \int_{0}^{t} d s e^{-\Gamma_{q}(t-s)} h(q) \sin (\mathbf{q} \cdot \Delta \mathbf{x}(s))\right\} \hat{\mathbf{r}} \frac{\partial W}{\partial r} .
\end{aligned}
$$

For a particle moving with constant velocity, $\Delta x=v_{0} s$; for this case the time integrals give

$$
\begin{aligned}
& \langle\mathbf{F}\rangle=c_{0} \int d \mathbf{r}\left[1+\frac{1}{(2 \pi)^{3}} \int d q h(q)\left\{\cos (\mathbf{q} \cdot \mathbf{R})\left[\exp \left(-\Gamma_{\alpha} t\right)+\Gamma_{q}\left(\frac{\Gamma_{Q} \cos \left(\mathrm{q} \cdot \mathrm{v}_{0} t\right)+\mathrm{q} \cdot \mathrm{v}_{0} \sin \left(\mathrm{q} \cdot \mathrm{v}_{0} t\right)-\Gamma_{Q} \exp \left(-\Gamma_{Q} t\right)}{\Gamma_{\alpha}^{2}+\left(\mathrm{q} \cdot \mathrm{v}_{0}\right)^{2}}\right)\right]\right.\right. \\
& \left.+\sin (q \cdot \mathbf{R}) \Gamma_{q} \frac{\Gamma_{q} \sin \left(q \cdot v_{0} t\right)-q \cdot v_{0} \cos \left(q \cdot v_{0} t\right)+q \cdot v_{0} \exp \left(-\Gamma_{q} t\right)}{\Gamma_{q}^{2}+\left(q \cdot v_{0}\right)^{2}}\right\} \hat{r} \frac{\partial W}{\partial r}
\end{aligned}
$$


Expanding $(\mathbf{q} \cdot \mathbf{R})$ in terms of $\mathbf{r}$ and $\mathrm{v}_{0} t$, integrating by parts on $r$, applying the approximation

$$
W=-K_{B} T\left(e^{-B W}-1\right),
$$

which is obviously valid for $|\beta W| \ll 1$, and using spherical symmetry to eliminate terms in $\sin (q \cdot r)$,

$$
\langle F\rangle=-\mathrm{v}_{0} \frac{c_{0} K_{B} T}{(2 \pi)^{3}} \int d \mathrm{q}\left(\mathrm{q} \cdot \hat{\mathrm{v}}_{0}\right)^{2} \frac{\left\lfloor h^{(2)}(q)\right]^{2} \Gamma}{\Gamma^{2}+\left(\mathbf{q} \cdot \mathrm{v}_{0}\right)^{2}}+A,
$$

where $A$ represents terms vanishing exponentially as $t$ $\rightarrow \infty$. If $\Gamma^{2} \gg\left(\mathrm{q} \cdot \mathrm{v}_{0}\right)^{2}$ and $\Gamma=K_{B} T q^{2} / f_{0}$, which approximations were also used in Ref. 4 ,

$$
\langle F\rangle=-\mathrm{v}_{0} \frac{c_{0} f_{0}}{(2 \pi)^{3}} \int d \mathrm{q}\left(\mathrm{q} \cdot \hat{\mathbf{v}}_{0}\right)^{2}\left[h^{(2)}(q)\right]^{2} .
$$

This form is the same as Eq. (6), which was, however, obtained by indirect means. The approximations used to obtain Eqs. (6) and (17), such as Eq. (15), do not appear to be identical in all respects.

\section{DIRECT INTERACTION AND $f_{m}$}

The procedure of Eqs. (7)-(17) is now applied to estimate the effects of direct interactions on the friction factor of the mutual diffusion coefficient. We consider explicitly the behavior of the dynamic structure factor

$$
S(\mathbf{k}, t)=\left\langle\sum_{i, j=1}^{N} N^{-1} \exp \left\{i \mathbf{k} \cdot\left[\mathbf{r}_{\imath}(0)-\mathbf{r}_{j}(t)\right]\right\}\right\rangle
$$

at small times, defining

$$
D_{m}=-\frac{1}{k^{2}} \lim _{t \rightarrow 0} \frac{d S(\mathbf{k}, t)}{d t}\lfloor S(\mathbf{k}, t)]^{-1},
$$

The limit $t \rightarrow 0$ is interpreted so that $t$ is always much greater than the Brownian velocity relaxation time $\tau_{B}$. The relation of $D_{m}$ to the quantity measured in the classical boundary-spreading measurement will not be considered here.

By dividing the velocity of each particle into an in- teractive part $\mathrm{v}_{I S}(t)$ and a Brownian part $\mathrm{v}_{B !}(t)$, one obtains to lowest order in time (see Appendix)

$$
\begin{aligned}
\lim _{t \rightarrow 0} \frac{d S(\mathbf{k}, t)}{d t}= & \frac{1}{N} \lim _{t \rightarrow 0}\left\langle\sum _ { i , j = 1 } ^ { N } \operatorname { e x p } [ i \mathbf { k } \cdot \mathbf { r } _ { i j } ( 0 ) ] \left[ i \mathbf{k} \cdot \mathrm{v}_{I i}(t)\right.\right. \\
& -\int_{0}^{t} d s \mathbf{k} \cdot \mathbf{v}_{B t}(s) \mathbf{k} \cdot \mathbf{v}_{B i}(t) \\
& \left.\left.-2 \int_{0}^{t} d s \mathbf{k} \cdot \mathbf{v}_{B i}(s) \mathbf{k} \cdot \mathbf{v}_{I t}(t)\right]\right\rangle
\end{aligned}
$$

The first line of Eq. (20) will be treated elsewhere ${ }^{6}$; it is found that in the absence of hydrodynamic interactions

$$
\begin{aligned}
\frac{1}{N} \lim _{t \rightarrow 0} & \left\langle\sum _ { t , j = 1 } ^ { N } \operatorname { e x p } [ i \mathbf { k } \cdot \mathbf { r } _ { i j } ( 0 ) ] \left[ i \mathbf{k} \cdot \mathbf{v}_{I t}(t)\right.\right. \\
& \left.\left.\quad-\int_{0}^{t} d s \mathbf{k} \cdot \mathrm{v}_{B i}(s) \mathbf{k} \cdot \mathbf{v}_{B i}(t)\right]\right\rangle=\left[\frac{-D_{0} k^{2}(1-\phi)}{S(\mathbf{k}, 0)}\right] S(\mathbf{k}, 0) .
\end{aligned}
$$

In this section the final term of Eq. (20), viz. ,

$$
\begin{aligned}
I= & \lim _{t \rightarrow 0} \frac{2}{N}\left\langle\sum_{i, j=1}^{N} \exp \left[i \mathbf{k} \cdot r_{i j}(0)\right]\right. \\
& \left.\times\left(-\int_{0}^{t} d s\left[\mathbf{k} \cdot \mathbf{v}_{B i}(s)\right]\left[\mathbf{k} \cdot \mathrm{v}_{I I}(t)\right]\right)\right\rangle
\end{aligned}
$$

is considered. [A term proportional to $\mathrm{k} \cdot \mathrm{v}_{I I}(s) \mathrm{k} \cdot \mathrm{v}_{I I}(t)$ is of second order in $t$ and will be neglected here. ]

The for ce on a probe is again obtained from Eq. (13); however, in this section $\Delta x(s)$ is assumed to arise from the random motions of the probe. The average \langle\rangle then includes an average over all possible paths $\Delta \mathbf{x}(t)$. $\mathrm{Re}-$ placing $R=\Delta x(t)+r$ and integrating by parts on $r$, Eq. (13) becomes

$$
\begin{aligned}
F(t)= & \frac{-c_{Q}}{(2 \pi)^{3}} \int d \mathrm{r} d \mathrm{q} k_{B} T h(q) \mathrm{q} \cos (\mathrm{q} \cdot \mathrm{r}) h(q)\left\{-\sin \left(\mathrm{q} \cdot \Delta \mathbf{x}_{t}\right)\left[\exp \left(-\Gamma_{q} t\right)+\Gamma_{q} \int_{0}^{t} d s\right.\right. \\
& \left.\left.\times \exp \left[-\Gamma_{q}(t-s)\right] \cos \left(\mathrm{q} \cdot \Delta \mathbf{x}_{s}\right)\right]+\cos \left(\mathrm{q} \cdot \Delta \mathbf{x}_{t}\right) \Gamma_{q} \int_{0}^{t} d s \exp \left[-\Gamma_{q}(t-s)\right] \sin \left(\mathbf{q} \cdot \Delta \mathbf{x}_{s}\right)\right\} .
\end{aligned}
$$

$\mathbf{r}$, being measured from the moving probe, is implicitly time dependent; terms in $\sin (q \cdot r)$ are eliminated by spherical symmetry in $r$. To proceed further, the approximation (15) is applied. Substitution of Eqs. (15) and (23) into Eq. (22) gives

$$
\begin{aligned}
I= & \frac{2}{N} \frac{c_{0} K_{B} T}{(2 \pi)^{3} f}\left\langle\sum_{t, j=1}^{N} \exp \left[i \mathbf{k} \cdot \mathbf{r}_{i j}(0)\right] \int_{0}^{t}\left(\mathbf{k} \cdot \mathbf{v}_{B i}\left(s_{1}\right)\right) d s_{1} \int d \mathrm{q} \mathbf{k} \cdot \mathrm{q}[h(q)]^{2}\right. \\
& \left.\times\left\{\Gamma_{q} \int_{0}^{t} d s \exp \left[-\Gamma_{q}(t-s)\right] \sin \left[\mathrm{q} \cdot\left(\Delta \mathbf{x}_{s}-\Delta \mathbf{x}_{t}\right)\right]-e^{-\mathbf{r}_{q} t} \sin \left(\mathbf{q} \cdot \Delta \mathbf{x}_{t}\right)\right\}\right\rangle .
\end{aligned}
$$

Equation (24) appears to be the simplest general form for $I$ for weak potentials. To effect a significant further reduction in Eq. (24), an explicit expression for $h(q)$ is needed. For particles with purely weak interactions, we use the Gaussian form

$$
\begin{aligned}
& h(q)=B_{0} r_{0}^{3} \exp \left(-q^{2} r_{0}^{2}\right), \\
& g(r)=1+\frac{B_{0} \exp \left(-r^{2} / 4 r_{0}^{2}\right)}{8 \pi^{3 / 2}},
\end{aligned}
$$


where $r_{0}$ and $B_{0}$ determine the range and strength of the potential, respectively. From Eqs. (24) and (26b), $W(0)$ $=-K_{B} T B_{0} /\left(8 \pi^{3 / 2}\right)$. Combining (26a) and (24), performing the angular part of $\int d q$, making an integration by parts on $|q|$ and replacing $\Gamma_{q}=D_{0} q^{2}$,

$$
\begin{aligned}
I= & \frac{-4 c_{0} K_{B} T B_{0}^{2} r_{0}^{6}}{(2 \pi)^{2} f}\left\langle\mathbf { k } \cdot \Delta \mathbf { x } ( t ) \left\{\int_{0}^{t} d s \frac{D_{0} \mathbf{k} \cdot \widehat{\Delta_{2} \mathbf{x}}}{\left|\Delta_{2} \mathbf{x}\right|^{2}}\right.\right. \\
& \left.\times \int_{0}^{\infty} d q \exp \left\{-q^{2}\left[D_{0}(t-s)+2 r_{0}^{2}\right]\right\}\left[5 q^{3} \sin \left(q\left|\Delta_{2} \mathbf{x}\right|\right)-2\left[D_{0}(t-s)+2 r_{0}^{2}\right] q^{5} \sin \left(q\left|\Delta_{2} \mathbf{x}\right|\right)\right]\right\} \\
& \left.\times \frac{-\mathrm{k} \cdot \widehat{\Delta \mathbf{x}}(t)}{|\Delta x(t)|^{2}} \int_{0}^{\infty} d q \exp \left[-\left(D_{0} t+2 r_{0}^{2}\right) q^{2}\right]\left[3 q \sin (q|\Delta \mathbf{x}(t)|)-2\left[D_{0} t+2 r_{0}^{2}\right] q^{3} \sin (\mathbf{q}|\Delta \mathbf{x}(t)|)\right]\right\rangle,
\end{aligned}
$$

where $\Delta_{2} \mathrm{x}=\Delta \mathrm{x}(s)-\Delta \mathrm{x}(t)$ and - indicates a unit vector. The integral on $q$ gives a confluent hypergeometric function. From Luke ${ }^{7}$ [Eq. 4.2(2)]

$$
\begin{aligned}
& { }_{1} F_{1}\left(\sigma, \nu+1,-\lambda^{2} / 4 z^{2}\right) \\
& \quad=\frac{2 \Gamma(\nu+1) z^{2 \sigma}}{\Gamma(\sigma)(\lambda / 2)^{2}} \int_{0}^{\infty} d u \exp \left(-z^{2} u^{2}\right) u^{2 \sigma-\nu-1} J_{\nu}(\lambda u) .
\end{aligned}
$$

Noting $\sin z=(\pi z / 2)^{1 / 2} J_{1 / 2}(z)$, and

$$
{ }_{1} F_{1}(a ; b ; z)=\sum_{n=0}^{\infty} \frac{(a)_{n} z^{n}}{(b)_{n} n !},
$$

where

$$
\begin{aligned}
& (a)_{n}=a(a+1) \cdots(a+n-1), \quad(a)_{0}=1, \\
& \int \exp \left(-z^{2} u^{2}\right) u \sin (\lambda u) d u=\frac{\sqrt{\pi} \lambda}{4 z^{2}} \exp \left(-\lambda^{2} / 4 z^{2}\right), \quad(30 \mathrm{a}) \\
& \int \exp \left(-z^{2} u^{2}\right) u^{3} \sin (\lambda u) d u=\frac{\sqrt{\pi} \lambda}{16 z^{7}}\left(6 z^{2}-\lambda^{2}\right) \exp \left(-\lambda^{2} / 4 z^{2}\right),
\end{aligned}
$$

$$
\begin{aligned}
& \int \exp \left(-z^{2} u^{2}\right) u^{5} \sin (\lambda u) d u \\
& =\frac{15 \sqrt{\pi} \lambda}{16 z^{7}}\left\{\exp \left(-\lambda^{2} / 4 z^{2}\right)\left[1-\frac{4}{3} \frac{\lambda^{2}}{4 z^{2}}+\frac{4}{15}\left(\frac{\lambda^{2}}{4 z^{2}}\right)^{2}\right]\right\} .
\end{aligned}
$$

If the potential $W$ is weak, the Brownian displacement during the period $(0, t)$ will be given in first approximation by its unperturbed form

$$
P(\Delta \mathbf{x}(s)-\Delta \mathbf{x}(t))=\left[4 \pi D_{0}(t-s)\right]^{3 / 2} \exp \left[\frac{(\Delta x(s)-\Delta x(t))^{2}}{4 D_{0}(t-s)}\right] .
$$

Equation (31) allows evaluation of the average over displacements, the reduction

$$
\langle\Delta \mathbf{x}(t):(\Delta \mathbf{x}(s)-\Delta \mathbf{x}(t))\rangle=-\left\langle[\Delta \mathbf{x}(s)-\Delta \mathbf{x}(t)]^{2}\right\rangle
$$

being used as needed. At this point the integration over $s$ is elementary.

$$
\begin{aligned}
& I=\frac{D_{0} k^{2} c_{0} B_{0}^{2} r_{0}^{3}}{24 \sqrt{2} \pi^{3 / 2}}\left[-4 y^{-1 / 2}+\frac{1}{6} y^{-3 / 2}+\left(x+\frac{3}{10}\right) y^{-5 / 2}\right. \\
& \left.+\frac{53}{15}+\pi-4 \arctan (\sqrt{y})\right] \text {, }
\end{aligned}
$$

In the limit $t \rightarrow \infty$,

$$
I=0.3917 \frac{D_{0} k^{2} S(\mathrm{k}, t) \phi_{T}|(g(0)-1)|}{24 \sqrt{2}},
$$

where $\phi_{T}=c_{0} V$ is the thermodynamic volume fraction of particles in the system, the "volume" $V$ of a Gaussian particle being defined:

$$
V=\left|\int d r(g(r)-1)\right|=4\left|B_{0}\right| r_{0}^{3}
$$

From Eqs. (19)-(21) and (33) the cross correlation between $v_{I i}(t)$ and $v_{B i}(s)(s \leq t)$ serves to retard the decay of $S(\mathbf{k}, t)$. Combining these equations, for weak Gaussian particles,

$$
D_{m}=\frac{K_{B} T}{f S(\mathrm{k}, 0)}\left[1-\phi_{H}-\frac{0.3917}{24 \sqrt{2}} \phi_{T}|g(0)-1|\right],
$$

$\phi_{H}$ being the hydrodynamic volume fraction of the solute. If $D_{m}$ is expressed formally as a ratio of thermodynamic, hydrodynamic, and kinematic factors as

$$
D_{m}=\frac{K_{B} T}{S(\mathbf{k}, 0)} \frac{1}{f_{m}}\left(1-\phi_{H}\right),
$$

to first order in concentration one may write

$$
\frac{1}{f_{m}}=\frac{1}{f}\left[1-\frac{0.3917}{24 \sqrt{2}} \phi_{r}|g(0)-1|\right] \text {. }
$$

Direct interactions therefore do serve to increase the effective drag coefficient in the mutual diffusion coefficient.

For weakly interacting particles, this effect is not large. Equations (17) and (26a) give the change in the Stokes' law drag coefficient $f_{s}$ for our model potential as

$$
f_{s}=f\left[1+\frac{\phi_{T}|g(0)-1|}{24 \sqrt{2}}\right]
$$

or

$$
\frac{f_{m}-f}{f_{s}-f}=0.39+0\left(\phi^{2}\right) .
$$

Direct interactions between diffusing particles are moderately less effective at hindering diffusion than at hindering very slow steady motion, at least in this system.

There is a classical argument, due originally to Einstein, ${ }^{8}$ that in the solvent-fixed reference frame $f_{m}$ should be identically the same as $f_{s}$. This argument is now understood to apply only as a long-wavelength, lowfrequency (i. e., $t \rightarrow \infty$ ) limit, in which the fluctuating forces relax in a time $\tau$ much shorter than the time $t$ of interest for diffusion. In the present case, the motion of the probe particle and the relaxation of the force fluctuations are governed by the same diffusion coeffi- 
cient $D_{0}$; requiring $t \gg \tau$ is effectively the same as requiring $D_{0} \ll D_{0}$, which is an absurdity. In other words, if encounters between diffusing macroparticles are taken into account, the low-frequency limit, needed if $f_{m}=f_{s}$ is to obtain, does not exist.

Weissman ${ }^{4}$ has suggested that direct interactions do not contribute to $f_{m}$. This argument is based on the assertion that the molecular mean potential (expressed as the chemical potential $\mu$ ) "depends on no variables which do not equilibrate rapidly with $c$. Rapidly means at a rate faster than $D_{m} k^{2}$..." Our result, however, is that the intermolecular forces contain terms (variables) which relax at rates which are not faster than $D_{m} k^{2}$, namely, there are terms which relax as $D_{m} q^{2}$ for $q \leq k$. Weissman's ingenious argument would therefore appear to fail because its underlying assumptions do not apply to the systems of interest here.

Schurr ${ }^{9}$ has previously obtained a result related to that of this paper. Using the Kirkwood form ${ }^{10}$ for the drag coefficient, he demonstrated that fluctuating polyelectrolyte-small ion interactions substantially enhance the friction factors of isolated, diffusing polyelectrolytes.

\section{APPENDIX: DEMONSTRATION OF EOS. (2) AND (20)}

This Appendix summarizes the justifications for the use of Eqs. (2) and (20), as will eventually be presented in full detail in Ref. 6.

The purpose of the prescription (2) is to replace a constrained integral, in which the integration is held to the $(3 N-1)$-dimensional hypersurface determined by Eq. (3), to an unconstrained integral over a $3 N$-dimensional space. In the unconstrained integral, the effect of the constraint is to be supplied by an additional factor in the kernel of the integral [the exponential on the righthand side of Eq. (2)]. The justification for (2) is that it is the simplest substitution which gives the correct answer when applied to the constrained integrals:

$$
\begin{aligned}
& \int_{a_{k 0}} d\{N\} \exp [-\beta(W-A)]\left[\sum_{i=1}^{N} \exp \left(\mathrm{i} k \cdot \mathbf{r}_{i}\right)\right]^{n}=a_{k 0}^{n}, \\
& \int_{a_{k 0}} d\{N\} \sum_{i=1}^{N} \exp \left(i \mathrm{q} \cdot \mathrm{r}_{i}\right) \exp [-\beta(W-A)]=0 \quad(\mathrm{k} \neq \pm \mathrm{q}),
\end{aligned}
$$$$
\int_{a_{k 0}} d\{N\} \exp [-\beta(W-A)] \sum_{i, j=1}^{N} \exp \left[i \mathrm{q} \cdot\left(\mathbf{r}_{i}-\mathbf{r}_{j}\right)\right]=N s(\mathbf{q})
$$

$$
(k \neq \pm q)
$$

(A1) follows from the definition of $\int_{a_{k 0}}$, while (A2) and (A3) are required by the statistical independence of $a_{k}$ and $a_{q}$ for $\mathrm{k} \neq \pm \mathrm{q}$. Equation (2) loses many mode-mode coupling effects, which are neglected consistently throughout this paper.
A previous paper of this author ${ }^{3}$ replaces the exponential with the product form

$$
\int d\{N\} \exp [-\beta(W-A)] \prod_{i=1}^{N}\left\lfloor 1+a_{k 0} \exp \left(-i \mathbf{k} \cdot \mathbf{r}_{i}\right)\right\},
$$

which lacks the self-terms $\exp \left(i \mathbf{k} \cdot\left(\boldsymbol{r}_{1}+\mathbf{r}_{\mathbf{i}}\right)\right)$ needed to satisfy $\mathrm{Eq}$. (A1) for $n>1$. The normalizing factor $\left\langle\left|a_{k}\right|^{2}\right\rangle$ in (2) has a physical interpretation, beyond the need for its presence in order to satisfy (A1). $a_{k}(0)$ may be increased either by changing the relative positions of the particles within clusters of nearby particles. The smaller $\left\langle\left|a_{k}\right|^{2}\right\rangle$ is, the harder it is to distort a cluster; as $\left\langle\left|a_{k}\right|^{2}\right\rangle$ becomes smaller, a given value of $a_{k}(0)$ corresponds more and more to the relocation of noninteracting particles.

Equation (20) is obtained from Eqs. (18) and (19) by first expanding (18) in terms of the displacements

$$
\mathbf{r}_{j}(t)-\mathbf{r}_{j}(0)=\int_{0}^{t} \mathbf{v}_{j}(s) d s
$$

and then decomposing $\mathbf{v}_{f}(s)$ into its Brownian and interactive parts $\mathrm{v}_{B j}(s)$ and $\mathrm{v}_{I j}(s)$, obtaining

$$
\begin{aligned}
S(\mathbf{k}, t)= & N^{-1} \sum_{i, j=1}^{N} \exp \left\{i \mathbf{k} \cdot\left[\mathbf{r}_{i}(0)-\mathbf{r}_{j}(0)\right]\right\} \\
& \times \sum_{n=0}^{\infty} \frac{\left[i \mathbf{k} \cdot \int_{0}^{t}\left[\mathbf{v}_{B i}(s)+\mathbf{v}_{t i}(s)\right] d s\right]^{n}}{n !} .
\end{aligned}
$$

One notes that

$$
\begin{aligned}
& \left\langle\mathrm{k} \cdot \mathrm{v}_{B i}(s)\right\rangle=0, \\
& \left\langle\int_{0}^{t} d s_{1} \int_{0}^{t} d s_{2} \mathrm{k} \cdot \mathrm{v}_{B i}\left(s_{1}\right) \mathrm{k} \cdot \mathrm{v}_{B i}\left(s_{2}\right)\right\rangle=2 D k^{2} t,
\end{aligned}
$$

and

$$
\mathrm{v}_{I i}(t)=\mathbf{F}_{i}(t) / f_{i},
$$

where $\mathbf{F}_{1}$ and $f_{1}$ are the force on $i$ and the drag coeffi cient of $i$, respectively. Taking $\lim _{t \rightarrow 0} d S(\mathrm{k}, t) / d t$, and observing that all terms containing two or more factors $\int_{0}^{t} d s$ vanish at $t \rightarrow 0$, a term-by-term expansion of the nonzero part of Eq. (A6) yields Eq. (20).

${ }^{1}$ R. Mazo, J. Chem. Phys. 43, 2873 (1965).

${ }^{2}$ D. Stigter, R. J. Williams, and K. J. Mysels, J. Phys. Chem. 59, 330 (1955).

${ }^{3}$ G. D. J. Phillies, J. Chem. Phys. 67, 4690 (1977).

${ }^{4}$ M. B. Weissman, J. Chem. Phys. 72, 231 (1980).

${ }^{5} \mathrm{~L}$. O. Onsager and R. M. Fuoss, J. Phys. Chem. 36, 2689 (1932).

${ }^{6}$ G. D. J. Phillies and P. R. Wills (submitted).

${ }^{7} \mathrm{Y}$. L. Luke, The Special Functions and Their Approximations (Academic, New York, 1969).

${ }^{8}$ A. Einstein, Ann. Phys. (Leipzig) IV 17, 549 (1905).

${ }^{9} \mathrm{~J}$. M. Schurr, Chem. Phys. 45, 119 (1980).

${ }^{10} \mathrm{~J}$. G. Kirkwood, J. Chem. Phys. 14, 180 (1946). 